\title{
Images and antibodies - The potential applications of phage antibody display to Clinical Microbiology
}

\author{
Roger Freeman \\ Public Health Laboratory, Institute of Pathology, \\ Newcastle General Hospital, Westgate Road, \\ Newcastle Upon Tyne, NE4 6BE, UK \\ E-mail:newrfree@north.phls.nhs.uk
}

\section{Introduction}

Clinical Microbiology is a very applied science, trying all the while to use any or all scientific techniques to achieve a very specific purpose: the rapid and accurate diagnosis of infections and infectious diseases. To this end, Clinical Microbiologists have quite unashamedly begged, stolen or borrowed methods and applications from anyone else! This endless plagiarism has left obvious footprints in the subject and has led to the often criticised "patchwork quilt" of approaches used in, for instance, the identification of species and typing of strains within them. Thus, Staphylococcus aureus is identified by an enzyme test (coagulase) and typed using bacteriophage susceptibility patterns, whereas Salmonellae are identified by antigen identification (of somatic or "O" and flagellar or "H" antigens) by serological means (the Kauffmann-White scheme) and Shigellae are identified by a combination of serological analysis and biochemical tests. More recently, molecular biology has been extensively applied (see later). In response to the inevitable and logical question as to why things are done in this empirical and unsystematic way, the answer is simply that they work, work well and, largely, work quickly. This short account advances the thought that phage antibody display (PAD) represents yet another opportunity for a raid by the Clinical Microbiologists and that it seems possible that PAD will offer some of the solutions required to achieve those desirable goals of speed, reliability and accuracy whilst at the same time additionally offering that other, very modern, aspiration of doing so at a low cost. It will be helpful first to examine the needs of Clinical Microbiology.

\section{The needs of Clinical Microbiology}

In addition to needing quick tests and accurate, reliable tests (both for obvious reasons) there is a growing need in Clinical Microbiology for non-cultural tests. These might be used on specimens in order to detect an organism or its product(s) as a means of establishing a clinical diagnosis or they might be used more quickly to identify an isolated organism or to confirm its expression of an important phenotypic characteristic, for instance, a toxin. There are two separate areas in which non-cultural tests might be usefully applied and, within each, several advantages of such an approach.

\subsection{Direct examination of clinical material}

Here the commonest problem is the presence of antibiotics in the specimen, making culture difficult or impossible. However, other reasons include diseases (e.g. tuberculosis) in which culture, even if successful, takes a very long time, the possibility of making retrospective diagnoses by detecting, in effect, the "footprints"of the organism, such as an antigen, long after the viable microbe is gone, the detection of so-called non-cultural viable (NCV) forms of microbes and the detection of small numbers of the target organism in a mixed flora of more robust organisms which may outgrow it in conventional culture. 


\subsection{Identification and differentiation of isolates}

The main problem here is the slow-growing organisms, such as mycobacteria. Conventional identification, relying on phenotypic characteristics, can take several weeks or more if subculture is necessary. In some species the inherent variability of an organism during subcultures can make conventional testing difficult whereas a non-cultural technique performed on the original isolate will more nearly reflect the nature of the isolate at the time it was first isolated.

In both these circumstances it should also be remembered that all cultures, even of those organisms classified as fast growers, is slow compared to non-cultural methods. Non-cultural methods might as often achieve the same purpose as culture, but quicker, as they might provide a novel form of identification.

\section{Current approaches to non-cultural methods}

Two approaches are in use.

\subsection{Molecular Biology}

The commonest method which employs molecular biology is PCR. Surprisingly to some, PCR is not necessarily very fast. One of the preferred approaches, especially in commercial kits, is PCR-ELISA. The attractions are that the technique can be semi-automated, that the ELISA step may increase sensitivity and that the ELISA stage can incorporate specific recognition of the amplicon, thus conferring specificity (an important safeguard in patient diagnostic work). In fact, many PCR-ELISA tests take up to 9 hours and can hardly be said to be "same day tests" in the common understanding of the term. PCR is certainly not cheap and even when performed in batches (itself a denial of the "quick test" concept) can attract costs of $£ 25-£ 60$. Furthermore, clinical material continue to throw up challenges which were not foreseen in research settings, especially the inability to extract only the target DNA instead of large amounts of unwanted, usually human, DNA within which might be the target. Substances inimical to the PCR reaction ("inhibitors") are frequent in clinical samples and often invalidate the test. Last, but not least, molecular methods provide genotypic answers - it is the phenotype which infects. Molecular approaches are excellent for rare conditions in which cost is not a major issue (e.g. meningitis), situations in which the clinical material is ordinarily sterile (blood,
CSF) and in which the target DNA is likely to be the only DNA (e.g. CSF, again) or in which the advantages of a rapid diagnosis are overwhelming, e.g. tuberculosis. Tuberculosis also offers a good example of the use of non-PCR molecular biology, in that most mycobacterial isolates are now identified by the use of gene probes, hybridising to RNA housekeeping genes. Such techniques have revolutionised mycobacterial identification, giving results within a few hours instead of several weeks. However, even here, some important distinctions to be made have so far eluded a molecular approach (see later).

\subsection{Immunological methods}

Immunological detection of organisms or their antigens has a much longer history in Clinical Microbiology than molecular techniques. Common examples include slide agglutination tests, ELISA tests and direct and indirect immunofluorescence. The antibodies used have previously been polyclonal and, therefore, of finite supply with time-consuming between-batch standardisation but more recently monoclonal antibodies have supplanted them. Such techniques are variously used to look for whole organisms (a good example being viruses), look for antigens, especially capsular polysaccharide antigens of organisms such as pneumococci or to detect expressed products of bacteria, for instance, toxins. These methods have been extremely successful. They are quick and relatively cheap. Nonetheless, there remain considerable problems with this approach.

Firstly, the target must, by definition, be antigenic, or, at least, immunogenic and preferably a protein. Preparing the antibodies is expensive, time-consuming and cannot avoid the use of animals. Additionally, most bacterial antigens are present in only minute amounts in their natural form. Recombinant technology can partly overcome this last problem but prokaryotic proteins are often over-expressed in expression systems and emerge as insoluble products. The necessary re-dissolving in such substances as urea often promotes an uncontrolled refolding so that the conformation of the final product may not totally reflect the natural substance. Finally, the commonest class of antigen of interest in bacteria, polysaccharides, are poor immunogens in any case.

These various points can be illustrated by three examples. 


\subsubsection{Tuberculosis}

The old axiom that if we could rapidly identify and treat all cases of infectious pulmonary tuberculosis the disease would die out in two generations remains true, even in the era of AIDS and multiply drug resistant tuberculosis (MDRTB). However, not all acid fast bacilli detected in the sputum by the cheap and rapid staining methods for such organisms (the Ziehl-Neelsen [ZN] and allied stains) are Mycobacterium tuberculosis. Consequently, it would be invaluable to devise a microscopic method which both detected the mycobacteria and, simultaneously, confirmed that they were $M$. $t u$ berculosis. TB PCR on sputum attempts this but only works reliably on specimens with a large number of the organisms. It is also expensive ( $£ 60$ versus the $40 \mathrm{p}$ for the Z-N stain) and thus precluded from use in the Third World where most of the disease occurs.

Another important goal is to identify quickly, cheaply but accurately the several different species which comprise the M. tuberculosis complex (MTBC). Present gene probes and PCR methods can only differentiate BCG from the others. The ability to distinguish between $M$. tuberculosis and M. bovis would be highly prized but it eludes molecular methods and the phenotypic methods take weeks and are not totally reliable. However, that this distinction is there to be made is undoubted. Both HPLC [1] and pyrolysis mass spectrometry (PyMS) [2,3] can make this distinction readily and reliably but neither method is widely available nor likely to be so.

\subsubsection{Haemophilus influenzae (HI) in bronchiectasis}

Bronchiectasis is a chronic chest condition in which the bronchi are distorted and the abnormal area acts as a reservoir for chronic infection. Bronchiectatics typically suffer repeated exacerbations of their chest infection but have long periods of quiescence and relatively good health between them. In an excacerbation the sputum is purulent and grows profuse Haemophilus influenzae within 18-24 hours. However, between exacerbations $\mathrm{HI}$ is also grown but in contrast to the periods of sputum purulence it takes $48+$ hours to grow. The genotype remains the same. The mechanism is thought to be a change in phenotype and specifically relates to iron utilisation and transferrin binding protein expression [4]. The ability rapidly and cheaply to detect the phenotype change would permit clinicians to prescribe antibiotics to pre-empt the onset of an exacerbation but also to withhold antibiotics in quiescence, thus limiting the chances of emerging resistance in the HI (a common problem). Clearly, molecular approaches are inappropriate and the distinction has, so far, eluded a conventional immunological approach.

\subsubsection{Pneumococcal disease}

Pneumococcus remains one of the principal pathogens in clinical microbiology, causing menigitis, otitis media, pneumonia and septicaemia. The mortality is often $30 \%$, although its predilection for the very young and the elderly often means that concomitant diseases make the prognosis particularly poor. Microbiologists need different non-cultural tests for these different settings. Thus, in the rare but life-threatening situations such as meningitis, cost is not an important consideration, the specimen (CSF) is ordinarily sterile and any DNA is likely to be that of the pneumococcus. This is, therefore, an ideal scenario for PCR and very sensitive and rapid real-time PCRs have been devised, targeting the pneumolysin gene [5]. They have the additional merit that they can also be adapted to detect the genes coding for penicillin resistance, which is an emerging problem in therapy of pneumococcal meningitis.

In chest infection and pneumonia, however, the situation is very different. Most large laboratories will process several hundred sputum specimens each month and the costs of PCR will be prohibitive, let alone the many problems associated with DNA extraction and inhibitors in this complex material with an abundant "normal" (i.e., non-pneumococcal) flora. In addition, there is a need to distinguish between carriage (the small numbers of pneumococci expectedly found in all respiratory secretions) and infection. Quantitative PCR would be even more expensive.

Non-cultural diagnosis of pneumococcal chest infection/pneumonia is currently attempted by the detection of capsular polysaccharide antigen (CPS) using antibodies coated onto inert particles, such as latex, or by countercurrent immunoelectrophoresis (CIE). Unfortunately, there are over 90 capsule serotypes to be catered for so the antiserum used (Omniserum) has to be polyvalent. Taken with the other problem that the target is a poor immunogen (a polysaccharide) it is not surprising that this test has a low sensitivity. There are also cross-reactions with some other (non-pneumococcal) streptococci found in the upper respiratory tract.

A logical solution to this problem is to develop a test to detect pneumolysin which is a species-specific, highly conserved thiol-activated toxin produced by all pneumococci. Its protein nature and high conservation make it an ideal target for monoclonal antibody production.

Following this strategy we have made several monoclonals and have demonstrated the benefits of this approach, using a Western blotting technique on sputum samples. The results showed that pneumolysin detec- 
tion (even by this relatively insensitive technique) was superior to CPS detection and was almost as sensitive as PCR [6]. A further theoretical advantage of pneumolysin detection is the observed fact that it accumulates in foci of pneumococcal infection and can be expected to distinguish between carriage and infection, certainly if its detection were to be quantified.

Unfortunately, the difficulties cited above in relation to the overexpression of prokaryotic proteins were experienced and it has proved impossible to translate the Western blotting into an ELISA format, probably, we believe, because the conformational epitopes of our recombinant did not faithfully reflect natural pneumolysin. We therefore urgently need to produce monoclonals (or their equivalent) to natural pneumolysin, but a 10L fermentation will yield only micrograms of this intracellular toxin.

\section{The attractions of phage antibody display technology}

The attractions of phage antibody display (PAD) in addressing the many problems discussed above are manifold. Its ability to recognise surface epitopes which might then allow us to distinguish between species and strains is valuable but the further dimension that the epitopes recognised need not be restricted to those which are antigenic or immunogenic (or even "self" and "not self" - a limitation which inevitably applies to animals) makes the approach potentially invaluable. Thus, in addressing the desired species distinctions to be made within the M. tuberculosis complex and which are known to exist but are beyond conventional immunology, PAD opens up a whole new approach. If this is successful, it is possible, in fact it is then extremely attractive to extend these studies to other mycobacterial species, comparing (in a panning approach), for instance, slow growers with fast growers, the MTBC with the other slow growers, etc. PAD could be a key to unlock the rapid identification of many mycobacterial species. The product, i.e. the phage(s) can then be adopted into a direct microscopic detection system, for instance, by incorporating fluorescein and the goal of detecting mycobacteria in sputum and confirming at the same time its speciation (see above) could be attained. Differentiation between species in cultures might be done by adopting the phage into an ELISA format. In this case a rapid and relatively inexpensive method could supplant both the slow phe- notypic methods and the rapid, but costly, genotypic methods.

Similarly, by using PAD to distinguish between two pneumococcal cultures which are (a) pneumolysin competent and (b) pneumolysin deficient, but which are otherwise isogenic, it should be possible to isolate phages which specifically recognise natural pneumolysin, thereby supplying the desired "monoclonal" to natural pneumolysin. The translation of pneumolysin-detection into a user-friendly ELISA format will then become a reality and provide an important new tool in the accurate and timely diagnosis of pneumococcal chest infection and pneumonia.

Again, a similar approach to HI, distinguishing between the two phenotypes described earlier would allow the development of a novel and useful test in the management of chronic chest disease.

Although this article cites only three examples of the potential benefits of PAD to clinical microbiology, it is certain that many more similar challenges occur which may be susceptible to this approach. The additional advantages of PAD (a relative short time to produce the reagent, a relatively inexpensive technology compared to the alternatives and, importantly, the removal of the need to use animals) serve only to emphasise the enormous attraction of this approach.

In conclusion, it seems that phage antibody display holds enormous promise of being a technology which can address many of the most desirable but frustratingly elusive goals of clinical, i.e. diagnostic, microbiology. As is the custom of clinical microbiologists, it is to be expected that PAD will be enlisted in their neverdiminishing effort to diagnose the infectious illnesses of human beings more quickly, more cheaply and more accurately.

\section{References}

[1] P.S. Duffey, L.S. Guthertz and G.C. Evans, Improved rapid identification of mycobacteria by combining solid-phase extraction with HPLC analysis of BACTEC cultures, J Clin Microbiol 34 (1996), 1939-1943.

[2] G. Wietn, J. Haverkamp, H.L. Meuzelaar, H.W. Engel and L.G. Berewald, Pyrolysis mass spectrometry: a new method to differentiate between the mycobacteria of the "tuberculosis complex" and other mycobacteria, J Gen Microbiol 122 (1981), 109-118.

[3] P.R. Sisson, R. Freeman, J.G. Magee and N.F. Lightfoot, Differentiation between mycobacteria of the Myocbacterium tuberculosis complex by pyrolysis mass spectrometry, Tubercle 72 (1991), 206-209.

[4] K.R. Hardie, R.A. Adams and K.J. Townner, Transferrinbinding ability of invasive and commensal isolates of Haemophilus spp, J Med Microbiol 39 (1993), 218-224. 
[5] A.M. Kearns, R. Freeman, O.M. Murphy, P.R. Seiders, M. Steward and J. Wheeler, Rapid PCR-based detection of Streptococcus pneumoniae DNA in cerebrospinal fluid, J Clin Microbiol 37 (1999), 3434.
[6] J. Wheeler, R. Freeman, M. Steward, K. Henderson, M.J. Lee, N.H. Piggott, G.J. Eltringham and A. Galloway, Detection of pneumolysin in sputum, J Med Microbiol 48 (1999), 853-866. 


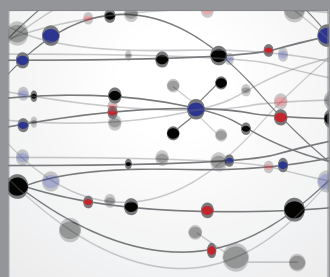

The Scientific World Journal
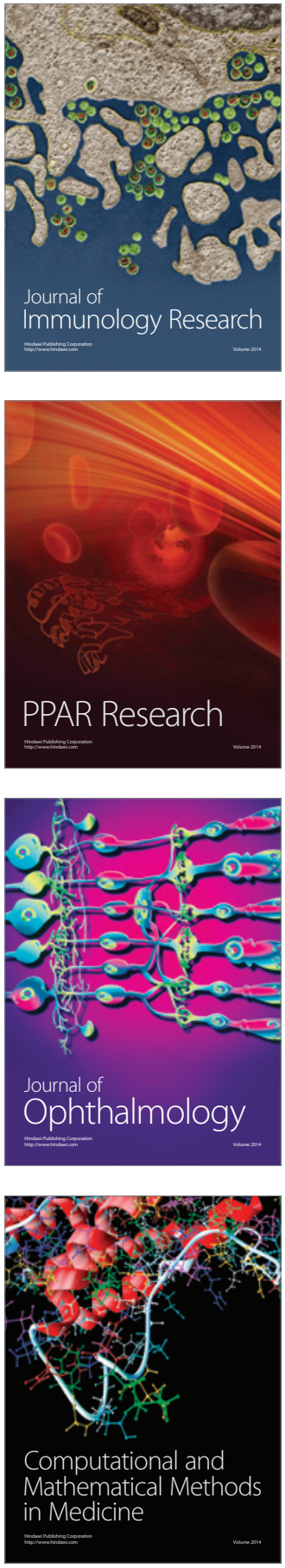

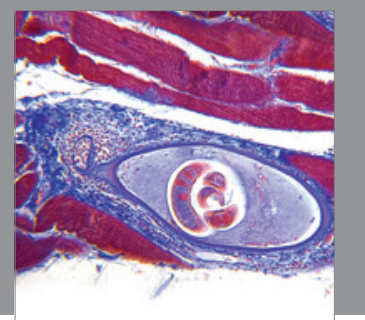

Gastroenterology

Research and Practice
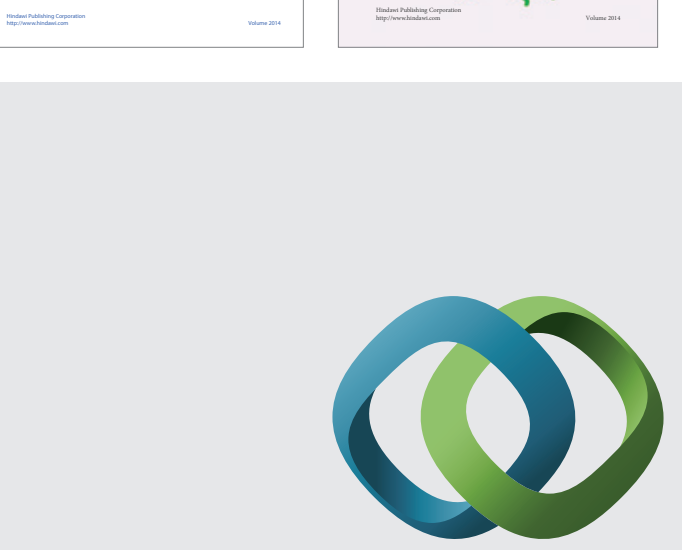

\section{Hindawi}

Submit your manuscripts at

http://www.hindawi.com
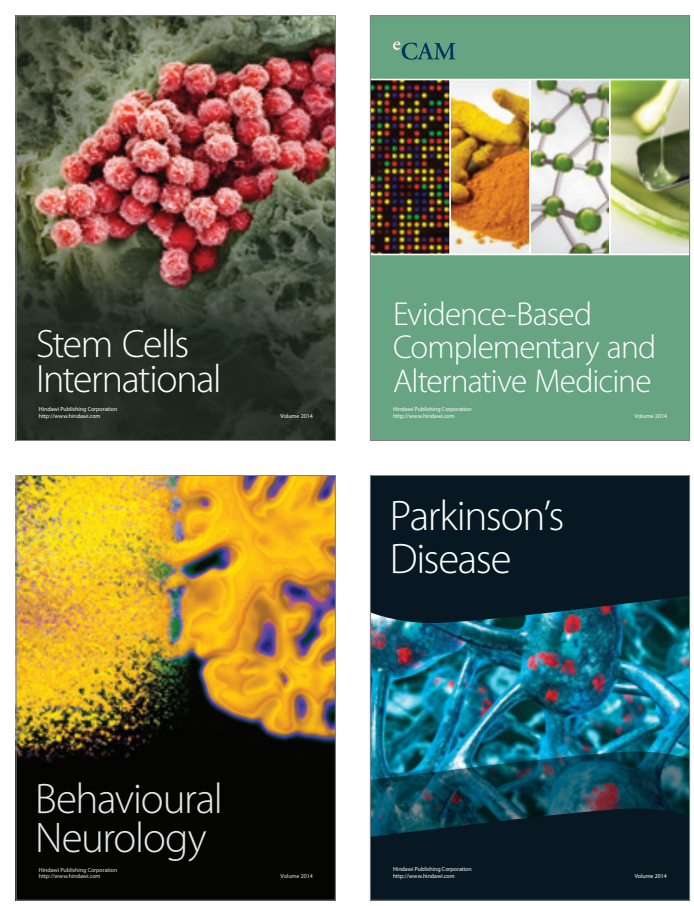

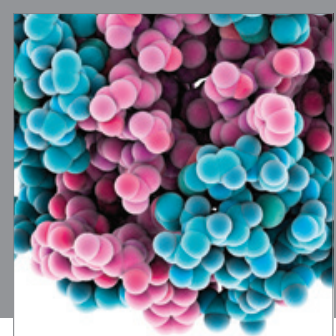

Journal of
Diabetes Research

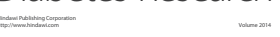

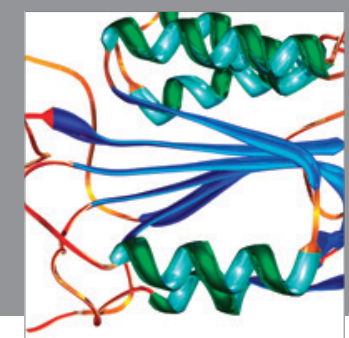

Disease Markers
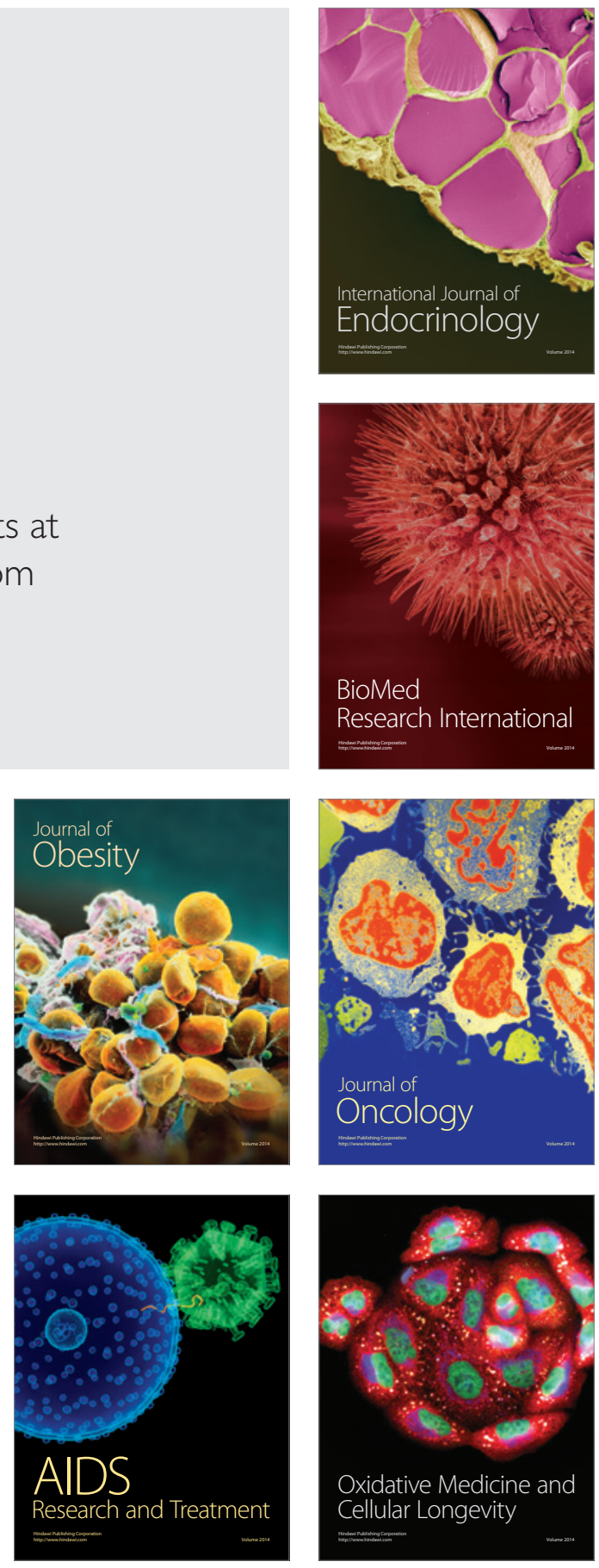